\title{
Fragmentos españoles de la Vulgata de 1-2 Macabeos: el fragmento de Vich y la Biblia de Valvanera; edición y estudio *
}

José Manuel CAÑAS REÍlLO

\section{INTRODUCCION}

El presente siglo ha sido extraordinariamente fecundo en estudios e investigaciones sobre las versiones latinas de 1-2 Mac ${ }^{1}$,

\footnotetext{
"Queremos expresar nuestro agradecimiento a algunas personas sin cuya ayuda este trabajo no se hubiera realizado: al Dr. H. J. Frede (m. 1998), director del Vetus Latina Institut de Beuron (Alemania), por las facilidades que nos dio para la consulta del fichero patrístico de este centro y por el envío de las fotografías del manuscrito de Madrid (Biblioteca de la Academia de la Historia) Aemilianensis 3, del Codex Hubertianus (Londres, British Museum, Add 24142) y del ms. de Oxford (Bodleian Library, Laud. Lat. 22); al Dr. Miguel dels Sants Gros, director del Museu Episcopal de Vich, por el envío de las fotocopias del fragmento de 1 Macabeos que se encuentra en ese museo.

${ }^{1}$ Las abreviaturas y siglas que utilizamos son las siguientes: $1-2 \mathrm{Mac}=1-2$ Macabeos; $\mathrm{BAH}=$ Biblioteca de la Academia de la Historia, Madrid; $\mathrm{BL}=$ Bodleian Library, Oxford; BNM = Biblioteca Nacional, Madrid; $B N P=B i-$ bliothèque Nationale, París; $\mathrm{BS}=$ Biblia Sacra iuxta Vulgatam versionem ... (Roma 1926ss.); ACV = Archivo Capitular de Vich; SMB = Seminario Mayor de Burgos; $\mathrm{UCM}=$ Universidad Complutense, Madrid; $\mathrm{v}=$ texto del fragmento de Vich; $\mathbf{V}=$ texto de Vulg de 1-2 Mac de la edición Libri I-Il Macchabaeorum, BS 18 (Roma 1995); Valv = Biblia de Valvanera; $V L=$ Vetus Latina, $\mathrm{VL}=$ Vetus Latina $:$ die Reste der altlateinischen Bibel (Friburgo $1949 \mathrm{ss}$. ); Vulg = Vulgata .

Usamos como texto de referencia el ya citado $\mathbf{V}$, así como las siglas con las que en esta edición son designados los mss. de $V u l g$. Con la sigla $\Lambda^{\mathrm{H}}$ designamos el texto de Vulg de 1-2 Mac del ms. de BAH, Aemilianensis 3 (no cotejado en BS 18), sobre nuestras propias colaciones. Usamos también el ms. $\Delta^{\mathrm{B}}$ del SMB, cotejado por D. DE BRUYNE - B. SODAR, Les anciennes traductions latines des Machabées,
} 
cuyo texto, tanto de $V L$ como de $V u l g$, es uno de los mejor conocidos de la Biblia latina en la actualidad. Ya en los siglos XVIII y XIX hubo estudios importantes sobre estos libros ${ }^{2}$, pero es realmente en 1932 cuando se inaugura el período brillante de estas investigaciones con la edición de D. De Bruyne y B. Sodar ${ }^{3}$, que

AM 4 (Maredsous 1932), pero no en Libri I-II Macchabaeorum. Para los testimonios de $V L$ seguimos la edición de DE BRUYNE - SODAR; para las glosas marginales de $V L$ de 1-2 Mac utilizamos nuestra edición Glosas marginales de Vetus Latina en Biblias vulgatas españolas: 1-2 Macabeos, TECC 65 (Madrid 2000). Los mss. de $V L$ son designados según la numeración de B. FISCHER, Verzeichnis und Sigel, VL 1 (Friburgo 1949). Las correspondencias de esas siglas son: $V L 7=\mathrm{BNP}, 11553 ; V L 95=$ Glosas marginales de $V L$ en el manuscrito de BAH, Aemilianensis 3, que fueron cotejadas muy deficientemente por DEBRUYNE - SODAR (utilizamos nuestra edición de las glosas marginales de $V L$ de 1-2 Mac ya citada); VL $109=$ UCM, 31;VL $146=$ Lyon, Bibliothèque Municipale 356 (430); VL 195 = Bolonia, Universidad 2571/628. Los tres primeros configuran en la edición de DE BRUYNE - SODAR el tipo textual $L$; el cuarto constituye el tipo $B$. Incluimos además lecturas del texto de $V L$ ( 1 Mac caps. 1-6) del Codex Hubertianus (British Museum, Londres, Add 24142), que pertenece al tipo textual $L$. Para las citas patrísticas seguimos el sistema de siglas y las ediciones recomendadas por H. J. FREDE, Kirchenschriftsteller: Verzeichnis und Sigel, VL 1/2 (Friburgo $1995^{4}$ ). Con la sigla $L X X$ designamos el texto griego de las ediciones de W. KAPPLER, Maccabaeorum liber 1, Septuaginta IX: Maccabaeorum libri I-IV, fasc. 1 (Gotinga $1967^{2}$ ), y R. HANHART, Maccabaeorum liber 2, Septuaginta IX: Maccabaeorum libri I-IV, fasc. 2 (Gotinga 1959).

${ }^{2}$ En el siglo XVIII, P. SABATIER, Bibliorum Sacrorum Latinae Versiones Antiquae, seu Vetus Italica, et caeterae quaecumque in codicibus MSS, et antiquorum libris reperiri potuerunt (Reims 1743; reimpr. Brepols, Turnhoult 1991) vol. II págs. 1013-1109, inaugura los estudios y ediciones de Vulg y VL de 1-2 Mac. En el siglo XIX destacan los de A. PEYron, M. T. Ciceronis Orationum pro Scauro, pro Tullio et in Clodium fragmenta inedita pro Cluentio, pro Caelio, pro Cecina, etc. Variantes lectiones. Orationem pro T. A. Milone a lacunis restitutam ex Membranis palimpsestis Bibliothecae R. Tuarinensis Athenaei. Edidit et cum Ambrosianis Parium orationum fragmentos componis Amedeus, Peyron (Stuttgart - Tubinga 1824) págs. 73117; A. M. CERIANI, «Liber Secundus Machabaeorum et Liber Tobiae cum aliorum fragmentis secundum ueterem latinam uersionem ex codice ambrosiano iterum editi», Monumenta Sacra et Profana 1/3 (Milán 1874) págs. 177-209; y S. BERGER, «Notice sur quelques textes latins inédits de l'Ancien Testament», NEMBN 34/2 (1893) págs. 147-152. A comienzos del siglo XX destacan los trabajos de G. MERCATI, «Frammenti Urbinati d'un'antica versione latina del libro II de'Maccabei editi ed illustrati da ...», $R B 11$ (1902) págs. 184-211; W. MOLSDORF, «Fragment einer altlateinischen Bibelübersetzung in der Königlichen und UniversitätsBibliothek zu Breslau», ZAW 24 (1904) págs. 240-250; y C. H. TURNER, «Iter Dunelmense: Durham bible mss. with the text of a leaf lately in the possession of Canon Greenwell of Durham, now in the British Museum», JTS 10 (1909) págs. 529-551.

${ }^{3}$ Les anciennes. 
tuvieron en cuenta todos los textos de $V L$ entonces conocidos y la primera edición crítica de la $V u l g$ de 1-2 Mac.

Pero con el paso del tiempo y los avances de la investigación, esa edición se ha revelado en algunos aspectos insuficiente. En 1969 bajo la dirección de R. Weber ${ }^{4}$ (con posteriores reediciones, entre las que destacan la tercera, dirigida por B. Fischer, y la cuarta, por R. Gryson) apareció una edición manual de la Vulg completa, que en algunos aspectos referentes a 1-2 Mac venía a completar a la de De Bruyne - Sodar para esa recensión. Dicha edición contribuye considerablemente a la reconstrucción del texto original de la Vulg respecto a la de De Bruyne - Sodar, pero su utilidad para el estudio de la transmisión de la Vulg resulta insuficiente. Tales carencias han sido solventadas con la publicación en 1995 del volumen correspondiente a 1-2 Mac de BS ${ }^{5}$, ya que se ha ampliado considerablemente el número de testimonios y familias de testimonios cotejados y es posible hacer un seguimiento de la historia de la transmisión de la Vulg de 1-2 Mac desde sus principios hasta el siglo $\mathrm{XV}^{6}$.

El estado en el que actualmente se encuentra la investigación sobre las versiones latinas de 1-2 Mac, especialmente de la Vulg, es el más propicio para la profundización en la historia de su texto tomando en consideración testimonios hasta ahora no cotejados. Esa es precisamente la finalidad del presente trabajo en el que nos hemos propuesto la edición y estudio del texto perteneciente a la Vulg de 1-2 Mac a partir de dos testimonios españoles hasta ahora inéditos, que presentan problemas diferentes:

1) Uno es el fragmento del Museo Episcopal de Vich, XIII/19, fol. 8. Hasta ahora no se ha cotejado en las ediciones de Vulg de

${ }^{4}$ R. Weber (dir.) et alii, Biblia Sacra iuxta Vulgatam versionem (Stuttgart 1969); $3^{\text {a }}$ ed. preparada por B. FISCHER, con la colaboración de H. J. FREDE, J. GRIBOMONT, H. F. D. SPARKS y W. THIELE (Stuttgart 1983); 4ª ed. por R. GRYSON (Stuttgart 1994).

${ }^{5}$ Libri I-II Macchabaeorum.

${ }^{6}$ Para una valoración de las aportaciones de esa edición cf. la reseña de P. M. BOGAERT en el Bulletin de la Bible Latine $7,2^{a}$ Ser. (1996) págs. 400-402. Además de la edición Libri I-II Macchabaeroum citada, hay que tener en cuenta otros estudios y ediciones sobre las versiones latinas de esos libros aparecidos después de la edición de DE BRUYNE - SODAR: F. VATTIONI, «Frammenti biblici latini», Augustinianum 15 (1975) págs. 389-422; H. J. FREDE, «Libri Macchabaeorum veteris latinae versionis», en Vetus Latina-Fragmente zum Alten Testament, H. J. FREDE U. FRÖHLICH - H. STANJEK (eds.), Aus der Geschichte der lateinischen Bibel 28 (Friburgo 1996) págs. 205-229; y CAÑAS REÍllo Glosas marginales. 
1-2 Mac citadas ningún testimonio de origen catalán y será interesante estudiar su texto y comprobar qué lazos de unión presenta con los restantes testimonios españoles y en qué medida tiene influencias de los textos franceses.

2) El otro es la Biblia de Valvanera, hoy perdida, algunas de cuyas variantes de Vulg se han transmitido en forma de glosas marginales en el incunable de Vulg de El Escorial (Biblioteca del Real Monasterio, 54.V.35). Esas glosas son muy pocas, pero al menos nos permitirán hacernos una ligera idea del tipo de texto de 1-2 Mac que tenía Valv, cuyo texto de Vulg la investigación contemporánea relaciona con la edición española $\Lambda$.

Así pues, el primer texto que vamos a estudiar nos dará una idea aproximada del tipo de texto que se copiaba en el escritorio de Vich en el siglo XI; el segundo nos permitirá hacer un sondeo en la historia de una parte de la tradición de la edición española $\Lambda$ en los siglos $\mathrm{X}$ al $\mathrm{XII}$.

\section{El FRAGMENTO DE VICH; EDICIÓN CRÍTICA}

El Museo Episcopal de Vich posee una importante colección de fragmentos manuscritos de la Vulg en la que está representada la mayor parte de los libros de la Biblia ${ }^{7}$. La importancia de esos fragmentos se debe a que son testimonios del texto de la Vulg utilizado en la región ausetana durante los siglos XI al XIII y a que, por pertenecer a un área geográfica fronteriza como es Cataluña, pueden ofrecernos valiosas informaciones sobre el intercambio de textos entre España y el resto de Europa en esa época. Uno de esos fragmentos, el XIII/19, fol. 8, contiene texto de Vulg de 1 Mac 1,46-2,12. Se trata de un folio de $33^{\prime} 5$ x $26^{\prime} 5 \mathrm{cms}$. $<25 \times 18$ cms.>, a dos columnas de 26 líneas, en minúscula carolina del siglo XI ${ }^{8}$. Lleva el número 21 en el catálogo de $\mathrm{M}$. dels Sants Gros de los fragmentos bíblicos del museo.

Como ya hemos dicho, aquí vamos a ocuparnos por primera vez de un testimonio de 1-2 Mac de esa procedencia geográfi$\mathrm{ca}$, pues hasta el momento en ninguna de las ediciones críticas

\footnotetext{
${ }^{7}$ Para estos fragmentos, cf. el catálogo de M. Dels SANTS Gros, «Fragments de Bíblies llatines del Museu Episcopal de Vic», RCatT 3 (1978) págs. 153-171.

${ }^{8}$ Seguimos la descripción de SANTS Gros «Fragments» pág. 165; para más detalles véase allí mismo. Para otros mss. de Vich, cf. T. Ayuso MARAzUela, La Vetus Latina Hispana: 1, Prolegómenos, TECC 1 (Madrid 1953) págs. 365-367.
} 
de Vulg de dichos libros se han cotejado los testimonios de origen catalán, como pueden ser la Biblia de Roda ${ }^{9}$ (BNP 6 , del siglo XI) y la erróneamente conocida como «Biblia de Farfa» (Roma, Biblioteca Apostolica Vaticana, Vat. lat. 5729, del siglo XI) que ya han sido objeto de múltiples estudios y ediciones para otros libros de la Biblia ${ }^{10}$, la Biblia de la Catedral de

${ }^{9}$ Sobre las biblias catalanas en general cf. D. DE BRUYNE, «Étude sur les origines de la Vulgate en Espagne», RBén 31 (1914-1919) págs. 373-401: pág. 376 nota 4.

${ }^{10}$ Ambos manuscritos han atraído la atención de los investigadores especialmente por sus elementos extrabíblicos que son de gran interés; la mayor parte de ellos ha sido cotejada en las ediciones de BS. Sobre el manuscrito de Roda (sigla R o R ${ }^{\circ}$ en BS) véanse: H. QUENTIN, Liber Genesis, BS 1 (Roma 1926); Libri Exodi et Levitici, BS 2 (Roma 1929); Libri Numerorum et Deuteronomii, BS 3 (Roma 1936); Libri Iosue, Iudicum, Ruth, BS 4 (Roma 1939); Liber Samuhelis, BS 5 (Roma 1944); Liber Malachim, BS 6 (1945); Liber Verborum Dierum, BS 7 (Roma 1948); Libri Ezrae, Tobiae, Iudit, BS 8 (Roma 1950); y Liber Danihelis, BS 16 (Roma 1981). De este manuscrito es especialmente interesante el Colofón a Ester, que sólo se encuentra además en otro testimonio (BNP, lat. 11553): E. MUNDó, «El colofó a Ester de la 'Pandectes minutiore manu' de Cassiodor», Miscellanea $B$. Ubach (Montserrat 1953) págs. 161-176; B. FISCHER, «Codex Amiatinus und Cassiodor", en B. FISCHER, Lateinische Bibelhandschriften im frühen Mittelalter, Aus der Geschichte der lateinischen Bibel 11 (Friburgo 1985) págs. 9-34 (reimpr. de BZ, N. F. 6 [1962] págs. 57-79) pág. 13; y W. THIELE, Sapientia Salomonis, VL 11/1 (Friburgo 1977-1983) pág. 95 nota 2. Del manuscrito del Vaticano (sigla F en BS) se han cotejado los elementos extrabíblicos en las ediciones citadas para el manuscrito de Roda, excepto en BS 1. Cf. además: B. FISCHER, «Bibelausgaben des frühen Mittelalters», en B. FISCHER, Lateinische Bibelhandschriften págs. 35-100 (reimpr. de La Bibbia nell 'alto medioevo, 26 aprile-2 maggio 1962, Settimane di studio del centro italiano di studi sull'alto medioevo 10 [Spoleto 1963] págs. 519 600); y P. SALMON, Les «Tituli Psalmorum» des manuscrits latins, CBL 12 (Vaticano 1959) págs. 134 y 138-148.

El texto de ambos manuscritos es de Vulg, a excepción de Tobit y $J$ udit, que en la Biblia de Roda son de $V L$, y de Hechos de los Apóstoles, que en esta misma Biblia es de $V u l g$ pero contiene numerosas lecturas de $V L$ en texto y margen. El texto de $V L$ de la Biblia de Roda es designado con el número 62 en FiSCHER Verzeichnis pág. 15. Sobre el texto de estos libros en los manuscritos citados cf. además: Novum Testamentum Domini nostri lesu Christi latine secundum editionem Sancti Hieronymi ad codicum manuscriptorum fidem recensuerunt, I. WORDSWORTH, olim episcopis Sarisburiensis et H. I. WHITE, olim aedis Christi decanus in operis societatem adsumtis, H. F. D. SPARKS, Sacrae Scripturae interpretationis Professore Orielensi et A. WhITE ADAMS, Sacrae Theologiae in Collegio Beatae Mariae Magdalenae decano, pars 3: Actus Apostolorum, Epistulae Catholicae, Apocalypsis Iohannis (Oxford 1954) pág. VII (sigla R para esta Biblia en Hechos); F. VATTIONI, «La Vetus Latina di Tobia nella Bibbia di Roda», RCatT 3 (1978) págs. $173-$ 
Urgel ${ }^{11}$ y el manuscrito 5 (LIX), fols. 105-135, del ACV ${ }^{12}$.

El texto bíblico de la Vulg utilizado en Cataluña en la Edad Media ha empezado a ser conocido muy recientemente, pero sólo respecto a algunos libros bíblicos entre los que no se cuentan 1-2 Mac. La primera hipótesis sobre el texto catalán se debe a S. Berger. En su opinión, Cataluña constituye un importante eslabón en la historia textual de la Vulg en España; esta historia tiene su punto de partida en Sevilla, pasa por Toledo y a través de Cataluña llega al sur de Francia ${ }^{13}$. En época de Carlomagno ese texto de $V u l g$ pudo haber penetrado ya en Francia y pudo haberse extendido por sus territorios, de forma que el texto de la Vulg utilizado en el Languedoc en el siglo XIII sería más catalán que languedociano ${ }^{14}$.

Tal hipótesis ha sido puesta en cuestión en los estudios y ediciones de la serie VL del Vetus Latina Institut de Beuron (Alemania). En los volúmenes de esta serie ${ }^{15}$ en que se han cotejado tes-

200; P. M. BOGAERT, «Recensions de la vieille version latine de Judith. II. Le Monacensis», RBén 85 (1975) págs. 241-265, y «Recensions de la vieille version latine de Judith. IV. Trois manuscrits et deux recensions», RBén 86 (1976) págs. 181-217; L. RosSO, «Una antica variante del libro de Tobit (Tob VII.9)», RSO 50 (1976) págs. 73-89; R. HANHART, Text und Textgeschichte des Buches Judith, $A A W G$, Phil. Hist. Kl., dritte Folge 109, MSU 14 (Gotinga 1949) pág. 14; J. H. PETZER, «Texts and Text Types in the Latin version of Acts», Philologia Sacra. Biblische und patristische Studien für Hermann H.J. Frede und W. Thiele zu ihrem siebzigsten Geburtstag, R. GRYSON (ed.), Aus der Geschichte der lateinischen Bibel 24/1 (Friburgo 1993) págs. 259-284: pág. 261 nota 10.

"P. Pujol Y TuBAu, "El manuscrit de la Vulgata de la Catedral d'Urgell», Butlletí de la Biblioteca de Catalunya 6 (1920-1922) págs. 98-145, con la publicación de algunas lecturas de 1-2 Mac en págs. 124-125.

${ }^{12} \mathrm{~J}$. GuDIOL, «Catàleg dels llibres manuscrits anteriors al segle XVIII del Museu Episcopal de Vich», Butlletí de la Biblioteca de Catalunya 6 (1920-1922) y 7-9 (1925) (reimpr. en J. GudIOL, Catàleg dels libres manuscrits anteriors al segle XVIII del Museu Episcopal de vich, amb un apéndix per E. Junyent [Barcelona 1934]).

${ }^{13} \mathrm{~S}$. BERGER, Histoire de la Vulgate pendant les premiers siècles du moyen âge (París 1893; reprod. facsímil de la edición de París 1893: Hildesheim-Nueva York 1976) págs. 25-26.

${ }^{14}$ BERGER Histoire pág. 77.

${ }^{15}$ H. J. FREDE, Epistula ad Philippenses et ad Colossenses, VL 24/2 (Friburgo 1966-1971), y Epistulae ad Thessalonicenses, Timotheum, Titum, Philemonem, Hebraeos, VL 25,1 (Friburgo 1975-1982) 25,2 (1983-1991); THIELE Sapientia, y de este mismo editor, Sirach (Ecclesiasticus), VL 11/2 (Friburgo 1987-); E. SCHULZFLÜGEl, Canticum Canticorum, VL 10/3 (Friburgo 1992-); U. FRÖHLICH, Epistulae ad Corinthios I, VL 22 (Friburgo 1995-); y H. S. EYMANN, Epistula ad Romanos, VL 21 (Friburgo 1996-). 
timonios de la recensión catalana (sigla $\kappa$ ), especialmente la Biblia de Roda $\left(\kappa^{\mathrm{P}}\right)$ y la del Vaticano $\left(\kappa^{\mathrm{V}}\right)$, se ha llegado a la conclusión, en contra de la opinión de S. Berger, de que el texto de la recensión catalana de la Vulg en los libros estudiados es producto de una mezcla de elementos textuales procedentes de diversas fuentes, en su mayor parte francesas.

Presentamos una edición crítica del texto v, seguida de un estudio de sus particularidades en relación con los restantes testimonios de Vulg cotejados en las ediciones al uso. Como procedimiento básico de edición hemos procurado mantener en lo posible las lecturas del manuscrito, que en general presenta un texto muy correcto. Nuestra intervención en el texto se limita a dos aspectos:

1) La normalización de la grafía, teniendo en cuenta las normas de la lengua latina y la época a la que puede pertenecer el texto, probablemente de antigüedad tardía (siglos V-VI ${ }^{16}$ ), aunque con una base textual mucho más antigua ( siglo IV?) con diversos retoques e influencias posteriores. No obstante, hemos sido muy conservadores en lo que se refiere a las grafías de los nombres propios, cuyas variantes suelen ser muy ilustrativas por la información que nos aportan sobre sus relaciones con otros testimonios latinos.

2) El desarrollo de las abreviaturas, que son las normales en los manuscritos de la época.

En la numeración de capítulos y versículos, de la que carece el manuscrito, seguimos las ediciones de Vulg. En lo referente a la puntuación, seguimos la que aparece en la recensión $V(V u l g)$ de la edición de De Bruyne - Sodar ${ }^{17}$ con la finalidad de ofrecer un texto legible. Hemos extendido el uso de mayúscula inicial a todos los nombres propios y referidos a Dios; el manuscrito generalmente no la presenta en tales casos.

\footnotetext{
${ }^{16}$ Según DE BRUYNe - SOdAR Les anciennes pág. XXXII, la confección de la revisión de Vulg de 1-2 Mac no es anterior al siglo V. Por otra parte, el texto Vulg de estos libros ya existía en el siglo VI, fecha en la que está datado el más antiguo testimonio que conocemos de él: el fragmento de Durham, Cathedral Library B. IV, 6, fol. 169*. Cf. TURNER «Iter Dunelmense» págs. 539-540, y Durham Cathedral Manuscripts to the End of the Twelfth Century, Introduced by R. A. B. MYNORS (Oxford 1939) pág. 14 n $^{\circ}$ 1; E. A. Lowe, English Uncial (Oxford 1960) pág. 17, $C L A n^{\circ} 153, y$ «A sixth century Italian Uncial Fragment of Maccabees and its eighth-century Northumbrian copy», Scriptorium 16 (1962) págs. 84-85; y Libri I-II Macchabaeorum pág. XI.

${ }^{17}$ Es la única edición que presenta puntuación en estos textos; las restantes ediciones citadas han publicado el texto per cola et commata y sin puntuación.
} 


\subsection{EL TEXTO}

[1]... ${ }^{146}$ Et misit rex Antiochus libros per manus nuntiorum in Hierusalem et in omnes ciuitates Iudae ut sequerentur legem gentium terrae, ${ }^{147}$ et prohiberent holocausta et sacrificia et placationes fieri in templo Dei, ${ }^{148}$ et prohiberent celebrari sabbatum et dies sollemnes, ${ }^{1 / 9} \mathrm{et}$ coinquinari sancta et sanctum populum Israel. ${ }^{150} \mathrm{Et}$ iussit aedificari aras et templa et idola et immolari carnes suillas et pecora communia, ${ }^{151}$ et relinquere filios suos incircunsisos, et coinquinari animas eorum in omnibus immundis et abominationibus, ita ut obliuiscerentur legem et immutarent omnes iustificationes Dei, ${ }^{152}$ et quicumque non fecissent secundum uerbum regis Antiochi morerentur. ${ }^{153}$ Secundum uerba haec scripsit omni regno suo, et praeposuit principes populi qui haec fieri cogerent; ${ }^{154} \mathrm{et}$ iusserunt ciuitatibus Iudae sacrificare. ${ }^{155} \mathrm{Et}$ congregati sunt multi de populo ad eos, qui dereliquerant legem Domini, et fecerunt mala super terram, ${ }^{156}$ et fugauerunt populum Israel in abditis et in absconditis fugitiuorum locis. ${ }^{157} \mathrm{Die}$ quinta decima mensis Casleu, quinto et quadragesimo anno aedificauit rex Antiochus abominandum idolum desolationis super altare Dei, et per uniuersas ciuitates Iudae in circuitu aedificauerunt aras, ${ }^{158} \mathrm{et}$ ante ianuas domorum et in plateis incendebant tura et sacrificabant, ${ }^{159} \mathrm{et}$ libros legis Dei combuserunt igni scindentes eos, ${ }^{160} \mathrm{et}$ apud quemcumque inueniebantur libri testamenti Domini et quicumque obseruabant legem Domini secundum edictum regis trucidabant eum. ${ }^{161}$ In uirtute sua faciebat haec populo Israel qui inueniebantur in omni mense in ciuitatibus. ${ }^{162} \mathrm{Et}$ quinta et uigesima die mensis sacrificabant super aram quae erat contra altare. ${ }^{163} \mathrm{Et}$ mulieres quae circumcidebant filios suos trucidabant secundum iussum regis Antiochi. ${ }^{164} \mathrm{Et}$ suspendebant pueros a ceruicibus per uniuersas domos eorum, et eos qui circumciderunt eos trucidabant. ${ }^{165} \mathrm{Et}$ multi de populo Israel definierunt apud se ut non manducarent immunda. Et elegerunt magis mori quam

1.46: nuntiorum] nunciorum; Hierusalem] ihrlm; Iudae] iudę; terrae] terrę.1.48: sollemnes] sollempnes.- 1.49: Israel] isrl-- 1.50: aedificari] hedificari; aras] haras; idola] ydola; immolari] imolari; pecora] peccora.- 1.51: relinquere] $u t$ uid.; incircuncisos ... animas] ut uid.; abominationibus] abhominationibus.- 1.53: haec $^{1}$ ] hęc; haec ${ }^{2}$ hęc.- 1.54 Iudae] iudę.- 1.56: Israel] isrl.- 1.57 aedificauit] hedificauit; abominandum] abhominandum; idolum] ydolum; Iudae] iudę; aedificauerunt] hedificauerunt; aras] haras $u t$ uid..- 1.58: et ante ... domorum] $u t$ uid..- 1.61 haec] hęc; Israel] isrl.- 1.62 aram] haram; quae] quę.- 1.63 quae] quę; iussum] scripsi, iussu.- $\mathbf{1 . 6 5}$ Israel] isrl. 
cibis malis coinquinari, ${ }^{166} \mathrm{et}$ noluerunt infringere legem dei sanctam, et trucidati sunt, ${ }^{167}$ et facta est ira magna super populum ualde.

[2] In diebus illis surrexit Matathias filius Iohannis filii Symeonis sacerdos ex filiis Ioharim ab Hierusalem et consedit in montem Modin. ${ }^{12} \mathrm{Et}$ habebat filios quinque: Iohannan qui cognominabatur Gaddis, ${ }^{13}$ et Simeon qui cognominabatur Tassi, ${ }^{14}$ et Iudas qui uocabatur Machabeus, ${ }^{15}$ et Eleazarus qui uocabatur Abaron, et Ionathas qui cognominabatur Affus. ${ }^{16} \mathrm{Hii}$ uiderunt mala quae fiebant in populo Iuda et in Hierusalem, $n$ et dixit Matathias: Uae mihi! ut quid natus sum uidere contritionem populi mei et contritionem ciuitatis sanctae, et sedere illic cum datur in manibus iniquorum? ${ }^{18}$ Sancta in manibus extraneorum facta sunt, templum eius sicut homo ignobilis, ${ }^{19}$ uasa eius captiua abducta sunt, trucidati sunt iuuenes eius in plateis, et iuuenes eius ceciderunt in gladio inimicorum. ${ }^{10} \mathrm{Quae}$ gens non hereditabit regnum eius et non obtinuit spolia eius? ${ }^{11} \mathrm{Omnis}$ compositio eius ablata est, quae erat libera facta est ancilla. ${ }^{12} \mathrm{Et}$ ecce sancta nostra et pulchritudo nostra et claritas nostra desolata ...

2.1: Hierusalem] irhlm.- 2.6: quae] quę; Hierusalem] irhlm.- 2.7: Uae] Ue; contritione $\mathrm{m}^{1}$ ] contricionem; contritione ${ }^{2}$ ] contricionem; sanctae] sanctę.-2.10: quae] quę; obtinuit] optinuit.- 2.11 quae] quę.- 2.12: pulchritudo] pulcritudo.

En el estudio del texto de $\mathrm{v}$ en relación con los restantes testimonios latinos utilizamos como referencia el texto $\mathbf{V}$. Teniendo en cuenta la relación de $\mathrm{v}$ con $\mathrm{V}$, hemos clasificado las lecturas en dos grupos: a) lecturas de $\mathbf{v}$ coincidentes con $\mathbf{V}, \mathrm{y}$ b) lecturas de $\mathbf{v}$ divergentes de $\mathbf{V}$.

\subsection{Lecturas de $v$ coincidentes con $\boldsymbol{V}$}

La mayor parte del texto $\mathrm{v}$ coincide casi exactamente con $\mathbf{V}$. El objetivo de la edición crítica de $\mathbf{V}$ ha sido la reconstrucción del texto más antiguo de $V u l g$ de estos libros, por lo que v estaría muy próximo a este tipo de texto. Entre los testimonios que parecen ser los mejores representantes de $\mathbf{V}$ se encuentran los manuscritos $\Pi^{\mathrm{G}} \mathrm{AM} \Phi \Omega$ y con ellos coincide casi completamente $\mathrm{v}$; esta coincidencia es especialmente extraordinaria entre v y $\Phi^{\mathrm{T} 2}$. A menudo el 
grupo de testimonios indicado tiene el apoyo de otros testimonios, como $\Theta S Z O$, y con ellos también concuerda $v$ frecuentemente. En estos casos v suele discrepar de los testimonios españoles $\left(C \Sigma \Delta \Lambda^{L}\right)$ e italianos $\left(\mathrm{Q} \Gamma^{\mathrm{A}} \Psi^{\mathrm{D}}\right)$.

Nos interesa especialmente en este aspecto resaltar la divergencia que hemos señalado de $\mathrm{v}$ con los testimonios españoles y su coincidencia con otros, especialmente franceses, porque viene a contradecir la hipótesis de S. Berger sobre el texto catalán de la $V u l g$, al menos en lo que se refiere a este testimonio. Ofrecemos algunos ejemplos de coincidencia de $\mathbf{v}$ con $\mathbf{V}$ y de discrepancia con algunos de los testimonios españoles que a veces tienen otros apoyos esporádicos:

1.46 in omnes $v \mathbf{V} /$ omnes $\mathrm{C} \Sigma \Delta \Lambda^{L}$

1.55 super terram $\vee \mathbf{V} /$ in terra $\mathrm{C} \Sigma \Lambda^{\mathrm{L}}$

1.60 Domini $^{2} \mathrm{vV} /$ om. $\Sigma$

1.64 ceruicibus $v \mathbf{V} /+$ earum $\Delta$

2.4 uocabatur $\mathrm{VV} /$ cognominabatur $\mathrm{C} \Sigma \Lambda^{\mathrm{L}} \Phi^{\mathrm{T}}$

2.6 Iuda $v \mathbf{V} / \operatorname{iud}(\mathrm{a}) \mathrm{e} \mathrm{C} \Sigma \Delta \Lambda^{\mathrm{L}}$

2.6 in Hierusalem $v \mathbf{V} /$ Hierusalem $C \Sigma \Delta \Lambda^{\mathrm{L}} \mathrm{Z}^{*} \mathrm{Q} \Gamma^{\mathrm{A}}$

2.9 iuuenes $^{1} \mathrm{vV} /$ senes $\Sigma \Delta \Lambda^{\mathrm{L}} \mathrm{M}^{2} \Phi^{\mathrm{T} 2 \mathrm{P}} \Theta^{\mathrm{M} 2} \Psi^{\mathrm{D}} \Omega$

Así pues, cuando $\mathrm{v}$ coincide con $\mathbf{V}$ presenta estrecha relación con testimonios como A (Amiatinus) y con otros, algunos de los cuales son de procedencia francesa. Probablemente v es representante del tipo de texto de Vulg estándar utilizado en toda Europa occidental durante los siglos XI-XIII (Biblias de la Universidad de París, sigla $\Omega$ ) representado especialmente por testimonios de las ediciones $\Phi \Theta$ y emanado de las reformas de Tours (siglos VIII-IX) y otros textos de esa época $(M)$.

\subsection{Lecturas de $\boldsymbol{v}$ divergentes de $\boldsymbol{V}$}

Las divergencias de $\mathrm{v}$ respecto a $\mathbf{V}$, muy poco numerosas, se pueden clasificar en dos grupos: omisiones y variantes.

\subsubsection{Omisiones}

Son dos y de poca importancia. En el primer ejemplo, v tiene el apoyo de otros testimonios latinos de Vulg y de un manuscrito de $V L ;$ en el segundo, v está aislado en la tradición textual latina: 
1.53 omnia uerba $\mathbf{V} /$ uerba $\mathrm{v}=\mathrm{M}^{*} \Omega^{\mathrm{J} 2} 7$

2.9 uasa gloriae $\mathrm{V} /$ uasa $\mathrm{V}$

En 1.53 encontramos de nuevo que $\mathrm{v}$ se relaciona con testimonios pertenecientes al área geográfica francesa, como son $M \Omega^{\mathrm{J}}$ y con el manuscrito de $V L 7$, que en 1 Mac presenta un tipo de texto procedente del sur de Francia.

\subsubsection{Variantes}

Constituyen el grupo más importante de discrepancias entre $\mathrm{v} y$ V. En estos casos v suele tener el apoyo de otros manuscritos, entre los que destacan los de procedencia continental (especialmente del grupo tardío $\Omega$ ), alguno español aislado y testimonios de $V L$. A menudo v tiene el apoyo de un texto griego subyacente particular. Ejemplos de ello son:

1.52 fecisset ... moreretur $\mathbf{V} /$ fecissent ... morerentur $\mathrm{v}=\Delta^{\mathrm{LM}^{*}}$. $\Pi^{\mathrm{G}} \mathrm{M} \Phi S Z^{2} \mathrm{O} \Omega$

1.61 faciebant $\mathrm{V} /$ faciebat $\mathrm{v}=\mathrm{A}^{*} \Phi^{\mathrm{T}^{*} \mathrm{RGP}} \Theta^{\mathrm{A}} \Psi^{\mathrm{D}^{*}}$

1.64 circumciderant $\mathrm{V} /$ circumciderunt $\mathrm{v}=\Pi^{\mathrm{G}} \mathrm{AM} \Phi \mathrm{Z}^{\text {2(ut uid.) }}$

2.1 in illis diebus $\mathrm{V} /$ in diebus illis $\mathrm{v}=\Pi^{\mathrm{G}} \Theta^{\mathrm{AM}} \mathrm{S} \Gamma^{\mathrm{A}} \Psi^{\mathrm{D}} \Omega 109146$ H 195 (cf. 7) LUC par $12(234,29)^{18}$, cum $L X X$

2.7 inimicorum $\mathrm{V} /$ iniquorum $\mathrm{v}=\Omega^{\mathrm{M}}$

2.8 in manu $\mathbf{V} /$ in manibus $\mathrm{v}=\Omega^{\mathrm{SJ} * \text { ut uid.) }}$

2.9 gladio $\mathrm{V} /$ in gladio $\mathrm{v}=\Sigma^{\mathrm{C}} \Lambda^{\mathrm{H}} \Pi^{\mathrm{G}} \Phi \Theta^{\mathrm{AM}} Z^{2} \Gamma^{\mathrm{A}} \Psi^{\mathrm{D}} \Omega 7109146$ H 195, cum $L X X$

2.10 hereditauit $\mathbf{V} /$ hereditabit $\mathrm{v}=\Lambda^{\mathrm{L}} \Phi^{\mathrm{G}} \mathrm{S}$

Como se puede ver por los ejemplos citados, incluso cuando $\mathrm{v}$ diverge de $\mathbf{V}$, se mantiene la coincidencia con el grupo $\Pi^{\mathrm{G}} \mathrm{M} \Phi \Theta S$ $\mathrm{ZO} \Omega$. A veces a este grupo se suman algunos testimonios aislados españoles, entre los que no se cuenta el mejor testimonio (C) y que en estos casos tienen presumiblemente lecturas que son producto de influencias extranjeras.

A veces, más esporádicamente, $v$ coincide con testimonios españoles en lecturas de gran interés, que pueden ser indicio de la existencia de algún estrato de esta procedencia en v:

\footnotetext{
${ }^{18}$ LUCífero de CAgliari, De non parcendo cum Deum delinquentibus, en Luciferi Calarilani opera quae supersunt, G. F. DIERCKS (ed.), CChr, Series Latina 8 (Turnhout 1978) págs. 195-261.
} 
1.56 effugauerunt $\mathrm{V} /$ fugauerunt $\mathrm{v}=\Delta$

2.5 cognominabatur ${ }^{1} \mathrm{~V}$ cum LXX $^{\mathrm{L}-19} 9346311 /$ uocabatur $\mathrm{v}=$ $C \Sigma \Delta^{L} 95109146$ H 195 , cum LXX ${ }^{\text {ed }}$

En 2.5 la lectura uocabatur de $\mathrm{vC} \Sigma \Lambda^{\mathrm{L}}$ tiene como Vorlage el texto griego mayoritario de $L X X$, frente a $\mathbf{V}$ que tiene como $V o r$ lage el texto griego luciánico. La lectura de $\mathrm{v}$, además, puede ser considerada como producto de la contaminación del texto de $\mathrm{Vulg}$ con $V L$, que suele ser una de las características de los testimonios españoles de Vulg en 1-2 Mac.

Del examen del texto de $\mathrm{v}$ en relación con los restantes testimonios de Vulg podemos concluir que:

a) El texto v es fiel representante del tipo textual de Vulg de 1 2 Mac utilizado prácticamente en toda Europa occidental en los siglos XI-XIII.

b) El texto v es más francés que español ${ }^{19}$. En efecto, se aprecia en la mayor parte de sus lecturas una clara influencia de las ediciones y textos relacionados con Tours, especialmente $\Phi \Theta$, y con el texto tardío estándar de la Universidad de París $(\Omega)$.

c) A pesar de ello, $v$ puede presentar un estrato textual que lo pone en relación con testimonios copiados y utilizados en España entre los siglos VII-X, en menor medida con $\mathrm{C}$, que es el mejor texto español, y en mayor medida con $\Sigma \Delta$, que a veces presentan contaminaciones de textos continentales.

3. LAS VARIANTES de la Biblia de VALVANERA EN EL INCUNABLE DE EL ESCORIAL

El incunable de $V u l g$ de la Biblioteca del Monasterio de El Escorial 54.V.35 (Venecia 1478) es conocido en el ámbito de estudios bíblicos porque contiene texto de las antiguas versiones latinas de la Biblia $(V L)$ en forma de glosas marginales ${ }^{20}$. Además,

${ }^{19}$ Cf. respecto a otros libros bíblicos estudiados en otros testimonios catalanes: SPARKS - WhITE Novum Testamentum pág. VII; THIELE Sapientia pág. 94; FREDE Ep. ad Thessalonicenses pág. 78; SchUlz-FLÜGEL Canticum pág. 62; y EYMANN Ep. ad Romanos pág. 60.

${ }^{20}$ Número de VL 94. La primera noticia sobre estas glosas se debe a $\mathbf{M}$. REVILLA, «El códice Ovetense de los Evangelios y la Biblia de Valvanera», $C D$ 117 (1919) págs. 393-399, 118 (1919) págs. 23-28 y 120 (1920) págs. 48-55 y 190-210 
contiene otra serie de glosas con variantes de Vulg ${ }^{21}$, aún poco conocidas y estudiadas, entre las que figuran algunas referentes a 1-2 Mac aún inéditas ${ }^{22}$, que son de las que aquí vamos a ocuparnos. Ambas series de glosas fueron copiadas en el incunable por $\mathrm{H}$. del Castillo en 1561 tomando como modelo Valv y el Códice Ovetense de los Evangelios del año $772^{23}$. Las de $V L$ se encontraban ya en forma de glosas en Valv, mientras que las de Vulg (encabezadas por la sigla $V$ ) se extrajeron del texto de esta Biblia.

Las glosas, tanto de $V L$ como de $V u l g$, son lo único que nos queda de Valv, que parece haberse perdido en el incendio ocurrido en la Biblioteca de El Escorial en 1671. Hasta el momento han resultado vanos todos los intentos de encontrarla, y sospechan algunos estudiosos que pudiera haberse salvado de la catástrofe como otros muchos manuscritos escurialenses, que fueron arrojados al exterior de la Biblioteca del Monasterio durante el incendio y en parte fueron a parar a la Biblioteca Universitaria de Upsala ${ }^{24}$.

(reimpr. Fragmenta Biblica Scurialensia: La Biblia de Valvanera y el códice Ovetense de los Evangelios [El Escorial 1920]). Este tipo de glosas se encuentra también en otros cinco manuscritos españoles que han sido objeto de múltiples ediciones y estudios. Cf. entre otros J. SCHILDENBERGER, Die altlateinischen Proverbien-Randlesungen der Bibel von Valvanera in der Vulgata Inkunabel 54.V.35 des Escorial, SFG 5 (Münster 1935); R. WEBER, Les anciennes versions latines du deuxième livre des Paralipomènes, CBL 8 (Roma 1945); T. AYUSO, «Una importante colección de notas marginales de la Vetus Latina Hispana», EstBíb 9 (1950) págs. 329-376 (reimpr. en AYUSo Prolegómenos págs. 409-436); B. FISCHER, Genesis, VL 2 (Friburgo 1951-1954); T. AYUSO, La Vetus Latina Hispana: 2, El Octateuco, TECC 6 (Madrid 1967); THIELE Epistulae Catholicae y Sapientia Salomonis; J. ZIEGLER, Randnoten aus der Vetus Latina des Buches lob in spanischen Vulgatabibeln, SAM (1980), Heft 2 (Múnich 1980); R. GRYSON, Esaias, VL 12 (Friburgo 1987-1997); C. MORANO RODRÍGUEZ, Glosas marginales de Vetus Latina en las Biblias Vulgatas españolas: 1-2 Samuel, TECC 48 (Madrid 1990); A. MORENO HERnÁNDEZ, Las glosas marginales de Vetus Latina en las Biblias Vulgatas españolas: 1-2 Reyes, TECC 49 (Madrid 1992); y CAÑAS REÍllo Glosas marginales.

${ }^{21}$ Las glosas de Vulg contienen variantes de Génesis, Éxodo, Levítico, Números, Jueces, 1-2 Samuel, 1-2 Reyes, 1 Crónicas, Proverbios, Sapienciales, 1-2 Mac, Evangelios y 1-2 Pedro (cf. Revilla Fragmenta pág. 53).

${ }_{22}$ M. Revilla publicó las glosas correspondientes a Génesis, Exodo, Números y NT.

${ }^{23} \mathrm{Cf}$. la nota del folio $1^{\mathrm{r}}$ del incunable, publicada por FisCher Genesis pág. 3*.

${ }^{24} \mathrm{~V}$. LUNDSTRÖM, «De codicibus graecis olim escorialensibus, qui nunc Upsaliae adservantur», Eranos $2 / 1$ (1897) págs. 1-7; S. TORRALlas TOVAR, «De codicibus graecis upsaliensis olim escurialensibus», Erytheia 15 (1994) págs. 191-258: págs. 191 ss. 
A pesar de su pérdida, gracias a las descripciones antiguas que nos han llegado de esta Biblia y al examen interno de las variantes que se nos han conservado, es posible, al menos en parte, hacernos una ligera idea de su configuración física y del tipo de texto de Vulg que contenía.

Respecto a la configuración de la Biblia, todos los datos que tenemos proceden de A. de Morales ${ }^{25} \mathrm{y}$ a este siguen todos los estudiosos modernos que han tratado de ella ${ }^{26}$. Sabemos que estaba compuesta por dos volúmenes en pergamino, contenía un Chronicon brevis per aetates ${ }^{27}$ y las Interpretationes de los nombres propios de la Biblia (tomadas de San Jerónimo, Liber Interpretationis Hebraicorum Nominum). En opinión de M. C. Díaz y Díaz, debía tratarse de un típico producto riojano, probablemente con la Cruz de los Ángeles al comienzo ${ }^{28}$. A. de Morales la dató en el siglo IX-X ${ }^{29}$; los autores modernos la datan en los siglos $\mathrm{X}^{30}$ o X-XI ${ }^{31}$.

Respecto a su contenido, esta Biblia debía de tener texto completo del AT y NT según Vulg y glosas marginales de $V L$ para algunos libros bíblicos. Para el conocimiento de su texto de Vulg sólo contamos con las informaciones que nos aportan las glosas de Vulg del incunable de El Escorial, algunas de las cuales editó y estudió M. Revilla. Según este autor, en el Pentateuco las glosas concuerdan con el códice Toledano (BNM, Vitr. 13-1 [Tol. 2-1], sigla de Vulg $\Sigma^{\mathrm{T}}$ ) y con el Codex Gothicus Legionensis (San Isi-

\footnotetext{
${ }^{25}$ Memoria de los libros que se deben tomar para el Real Monasterio de San Lorencio, de los que tenía el Obpo. de Plasencia Don Pero Ponce de León, ms. Esc. \&-II 15, fol. 238v, y Coronica General de España (Córdoba 1586) tomo III págs. 329-330.

${ }^{26}$ Revilla Fragmenta pág. 3; T. Ayuso, «Los elementos extrabíblicos de la Vulgata», EstBíb 2 (1943) págs. 133-187: págs. 166ss, y Prolegómenos pág. 357; FISCHER Genesis págs. 2*-4*; y M. C. DÍAZ Y DİAZ, Libros y librerias en la Rioja altomedieval (Logroño 1979) págs. 93-94.

${ }^{27}$ Sobre el Chronicon en especial y los problemas de datación que presenta, cf. L. VÁzQUez de PARGA, «La Biblia en el reino astur-leonés», en La Bibbia nell'alto medioevo, 26 aprile-2 maggio 1962, Settimane di studi del Centro Italiano di Studi sull'alto medioevo 10 (Spoleto 1963) págs. 257-280 y discusión en págs. 337-338.

${ }^{28}$ Libros págs. 93-94.

${ }^{29}$ Coronica pág. 330.

${ }^{30}$ Ayuso Prolegómenos pág. 357; FISCHER Genesis pág. 3*. 811.

${ }^{31}$ Thiele Sapientia pág. 21, y Ep. Catholicae pág. 17*; y Gryson Esaias pág.
} 
doro de León, 2, sigla de $V u l g \Lambda^{\mathrm{L}}$ ); a veces parecen derivarse de la $V L$, y las del NT se corresponden con la edición de WordsworthWhite ${ }^{32}$. Más recientemente B. Fischer ${ }^{33}$ ha señalado la pertenencia de $V a l v$ a la edición española $\Lambda$, acabada por Florencio quizá en el año 940 en el Monasterio de Valeránica (Burgos) ${ }^{34}$.

Esto es todo lo que sabemos sobre Valv. En lo que se refiere especialmente a las variantes de Vulg es necesario revisar las conclusiones a las que llegó M. Revilla sobre su relación con otros testimonios latinos, dado que ahora disponemos de mejores ediciones críticas y conocemos mejor la historia de la transmisión de la Vulg.

Vamos a centrarnos seguidamente en la edición y estudio del texto de 1-2 Mac de las glosas de Vulg de nuestro incunable. Sólo tiene seis glosas de esos libros ${ }^{35}$; material muy exiguo, pero es de lo único que disponemos para el conocimiento de algunas de las características del texto de 1-2 Mac de Valv o al menos para hacernos una ligera idea del lugar que ocupa Valv en el ámbito de la tradición textual española de la Vulg.

\footnotetext{
${ }^{32}$ Novum Testamentum, 4 vols. (Oxford 1889-1954).

33 Vid. «Bibelausgaben» págs. 72-73 y 75-76 para los argumentos en favor de la cronología tardía de esta edición.

${ }^{34}$ El grupo $\Lambda$ está formado por los testimonios que a continuación se señalan (seguimos a FISCHER «Bibelausgaben» págs. 72-73). 1) San Isidoro de León, Codex Gothicus Legionensis ( $\mathrm{n}^{\circ}$ de $V L$ 133; glosas marginales de $V L, \mathrm{n}^{\circ}$ de VL 91; sigla de $V u l g \Lambda^{\mathrm{L}}$ ), copiada en el Monasterio de Valeránica (Burgos) o en todo caso en Castilla en el año 960. 2) Biblia de Oña, de la que quedan 11 folios en la Casa Central de la Hermandad de los Sacerdotes Operarios y 1 folio en el Archivo de la Abadía de Silos, con texto de los Evangelios (cf. A. ANDRÉs, «Oña: fragmento de la Biblia visigoda del s. X», Boletín de la Comisión Provincial de Monumentos Históricos y Artísticos de Burgos 76 (1941) págs. 575-581; y T. Ayuso, La Biblia de Oña: Notable fragmento casi desconocido de un códice visigótico homogéneo de la Biblia de San Isidoro de León [Zaragoza 1945]); fue terminada por Florencio, maestro de Sancho, en 943 en Valeránica. 3) San Isidoro de León, cod. 1.3 (glosas marginales de $V L, \mathrm{n}^{\circ}$ de VL 92); es copia directa del Codex Gothicus. 4) BAH, Aemilianense 2-3, siglo XIII; procede de San Millán de la Cogolla (glosas marginales de $V L, \mathrm{n}^{\circ}$ de VL 95; sigla de $V u l g \Lambda^{\mathrm{H}}$ ). 5) Catedral de Calahorra, 2 (glosas marginales de $V L, \mathrm{n}^{\circ}$ de VL 96); copiada en 1183 en Calahorra, quizá copia de Valv, y de la que sólo se conservan partes del primer volumen. Y 6) Cabildo de Lérida, quizá copiada en Calahorra en los siglos XII-XIII.

${ }^{35}$ Hemos cotejado las glosas directamente sobre el incunable y, en efecto, hemos podido constatar que son seis y no cuatro como se afirma en Libri I-II Macchabaeorum pág. XIX nota 8, donde se hace referencia sólo a las glosas de 1 Mac 2,42, 3,20 y 5,64, y $2 \mathrm{Mac} 2,11$. Las otras dos, que van encabezadas por la sigla $V$ en el incunable, han sido publicadas por los editores de la edición citada entre las de $V L 94$.
} 
Las glosas de Valv se refieren a 1 Mac 2,42, 3,20 y 5,64, y 2 Mac $2,11,12,15$ y 14,20 . Tres de ellas (1 Mac 2,42, 5,64 y 2 Mac 2,11) van acompañadas de glosas de $V L$ que reproducen el mismo pasaje en formas textuales próximas al tipo textual $L^{36}$. Como ya hemos dicho antes, todas las glosas de Vulg van encabezadas en el incunable por la sigla $V$, que verosímilmente señala su pertenencia a Vulg. El criterio de selección de estas perícopas por H. del Castillo para su conversión en glosas ha sido probablemente la discrepancia con el texto de Vulg del incunable escurialense, en relación a cuyo texto están copiadas en los márgenes. Pero no podemos estar seguros de hasta qué punto este criterio ha sido rigurosamente seguido por $\mathrm{H}$. del Castillo y por tanto no podemos suponer que el texto restante de Valv hubiera sido similar al del incunable y por ello no se hubieran incluido otras variantes. Seguramente debía de haber muchas más diferencias entre ambos textos, pero las recogidas en las glosas pueden haber sido las más llamativas para $H$. del Castillo.

\subsection{El texto}

Editamos el texto de Valv manteniendo sus particularidades gráficas y sus faltas de copista. Hemos prescindido de las siglas de encabezamiento, que en todos los casos, como ya hemos dicho, es $V$. Así pues, esto es lo que queda del texto de $V u l g$ de 1-2 Mac de Valv:

\section{Mac}

2,42 synagoga ad auxilium eorum

3,20 superbiae

5,64 faustos

\section{Mac}

2,11 mundatum 12,15 uelociter 14,20 cum iudeis

Vamos a estudiar detalladamente cada una de estas glosas, intentando establecer sus lazos de unión con los restantes testimonios latinos. Hacemos frecuente referencia también a $L X X$, de la que todas las versiones latinas de 1-2 Mac son traducción muy literal, ya que puede contribuir a iluminar algunos problemas textuales de la tradición textual latina. Para los testimonios latinos de $V L$ y de Vulg que aparecerán, remitimos a las ediciones ya cita-

\footnotetext{
${ }^{36}$ Cf. la edición de DE BRUYNE - SODAR Les anciennes.
} 
das. A los testimonios de Vulg ya editados sumamos el manuscrito de BAH, Aemilianensis 2-3, de los siglos XII-XIII (sigla de Vulg, $\Lambda^{\mathrm{H}}{ }^{37}$, también perteneciente a la edición $\Lambda$, que, como más adelante veremos, tiene una gran importancia para nuestro estudio. Aún no ha sido cotejado para 1-2 Mac, pero es el testimonio de $\Lambda$ más estudiado (después del Codex Gothicus Legionensis ${ }^{38}$ ) en las ediciones BS ${ }^{39}$ y VL ${ }^{40}$ de otros libros bíblicos. Lo hemos cotejado sobre fotografías.

1 Mac 2,42: synagoga ad auxilium eorum; $L X X: \sigma v v \alpha \gamma \omega \gamma \grave{\eta}$

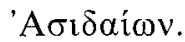

${ }^{37}$ Sobre este manuscrito, cf. H. QUENTIN, Mémoire sur l'établisement du texte de la Vulgate: Irc partie, Octateuque, CBL 6 (Roma-París 1922) págs. 300 y 327-330 y Liber Genesis pág. XVI; AYUSO La Vetus Latina: 1, Prolegómenos págs. 369-370; FISCHER Genesis págs. 4*-5*; FREDE Ep. ad Ephesios pág. 22*, Ep. ad Philippenses pág. 21, y Ep. ad Thessalonicenses pág. 65; THIELE Ep. Catholicae pág. 32*, Sapientia pág. 70-71, y Sirach pág. 49; W. CAHN, La Bible Romane, Chefs-d oeuvre de l'enliminure (Friburgo 1982) pág. 292; MORANO RODRÍGUEZ Glosas marginales pág. XXX; MORENo HeRnándeZ Las glosas marginales págs. 34-35; FröHLICH Ep. ad Corinthios $I$ pág. 91; además, sobre su texto de Vulg, cf. H. J. Frede, Pelagius, der irische Paulustext, Sedulius Scottus, Aus der Geschichte der lateinischen Bibel 3 (Friburgo 1961) pág. 81; FISCHER «Bibelausgaben» págs. 73 y 75-76; W. THIELE, Die lateinischen Texte des 1. Petrusbriefes, Aus der Geschichte der lateinischen Bibel 5 (Friburgo 1965) pág. 130; y H.J. FrEDE, «Ein Paulustext aus Burgund», Bib 54 (1973) págs. 516-536.

${ }^{38}$ Cf. sobre este manuscrito J. PÉrez Llamazares, Catálogo de los Códices y documentos de la Real Colegiata de San Isidoro de León (León 1923) págs. 4-18; QUENTIN Mémoire pág. 300 y Liber Genesis págs. XIV-XV; AYuso La Vetus Latina: I, Prolegómenos págs. 354-355; P. GaLinDo, «La 'Biblia de León' del 960», SFG 16 (Münster 1960) págs. 37-76; T. AYUSO, «La Biblia Visigótica de San Isidoro de León», EstBíb 19 (1960) págs. 5-24, 167-200 y 271-309, y 20 (1961) págs. 5-41,243-259 y 359-406; FREDE Ep. ad Thessalonicenses pág. 65; THIELE Sapientia págs. 71-72, Sirach pág. 49 y Ep. Catholicae págs. $17 *-18 *$ y $32 *-33 *$; ScHULZFLỨGEL Canticum págs. 51-52; FRÖHLICH Ep. ad Corinthios I págs. 92-93; EYMANN Ep. ad Romanos pág. 48. Un estudio amplio y detallado, centrado fundamentalmente en el aspecto textual de este ms. y sus relaciones con los demás mss. españoles de Vulg lo encontramos en B. FISCHER, «Algunas observaciones sobre el Codex Gothicus de la R. C. de S. Isidoro de León y sobre la tradición española de la Vulgata», ALeo 29-30 XV (1961) págs. 5-47.

${ }^{39}$ QuENTIN Liber Genesis. En los restantes volúmenes su texto ya no fue tenido en cuenta por considerarlo muy similar a $\Lambda^{\mathrm{L}}$ y porque contiene muchas lecturas tardías. Los elementos extrabíblicos de este manuscrito, sin embargo, han sido cotejados en la mayor parte de los volúmenes de BS.

${ }^{40}$ En la serie VL el texto de $\Lambda^{H}$ ha sido cotejado en la mayor parte de los volúmenes: Thiele Ep. Catholicae, Sapientia Salomonis y Sirach (Ecclesiasticus); FREDE Ep. ad Ephesios y Ep. ad Thessalonicenses; SCHULZ-FLÜGEL Canticum; FRÖHLICH Ep. ad Corinthios I; y EYMANN Ep. ad Romanos. 
Esta lectura es claramente una falta de copista, probablemente en lugar de la forma correcta Asideorum (V), en consonancia con $L X X$. Esta misma falta de copista se encuentra en $\Lambda^{H}$ y con algunas diferencias (in auxilia eorum) en el manuscrito de $V L 146$, que es el representante principal de la recensión $L$ en la edición de De Bruyne - Sodar. El término griego 'A $\sigma \_\alpha i \omega v$ debió de crear dificultades a los traductores y revisores latinos ${ }^{41}$, que optaron por tres vías para su traslación al latín:

a) Transliteración: $A \operatorname{sid}(a)$ eus ${ }^{42}$, que es la lectura de $\mathbf{V}$ y del $\mathrm{ms}$. de $V L \mathrm{H}$ (recensión $L$ ).

b) Armonización con el contexto, probablemente a partir de una trascripción a partir del griego existente previamente en latín, lo que ha tenido como resultado faltas de transmisión como las de $\Lambda^{\mathrm{H}}$ y 146, o el desconcertante Asiaticorum que encontramos en el manuscrito de $\mathrm{BL}{ }^{43}$, Laud. Lat. 22 , que presenta texto según la recensión $B$ de De Bruyne - Sodar ${ }^{44}$.

c) Omisión, como en una cita de LUC par 9,177,3.

La restante tradición textual latina presenta la traducción $I u$ daeorum, con una parte de la tradición textual de $L X X$.

\section{Mac 3,20: superbiae; $L X X$ : $\dot{\alpha} v o \mu i a \varsigma$}

El contexto de $\mathbf{V}$ al que pertenece este término es: Ipsi ueniunt ad nos in multitudine contumaci et superba ut disperdant nos et

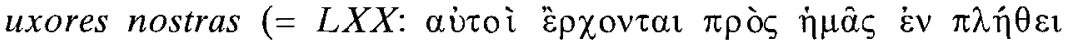

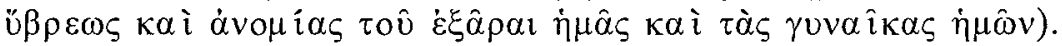
Superbiae sólo aparece en Valv y $\Lambda^{\mathrm{H}}$, pero presenta afinidades menos relevantes con otros testimonios latinos:

\footnotetext{
${ }^{41}$ Sobre este término cf. F. M. ABEL, Les Livres des Macchabées, EB (París 1949) pág. 43; J. A. GoldSTEIN, I Maccabees, A New Translation with Introduction and Commentary, AB 41 (Nueva York 1984²) págs. 5-6; y K. D. SCHUNCK, Historische und legenderische Erzählungen: I, Makkabäerbuch, JSHRZ 1,4 (Guitersloh 1980) pág. 306.

${ }^{42}$ El término Asidaeus está atestiguado en latín en el texto de Vulg de 1 Mac 2,42 y 7,13, y 2 Mac 14,6; cf. J. PERIN, Onomasticon Totius Latinitatis (Patavii 1913) vol. I pág. 190; Thesaurus Linguae Latinae vol. II pág. 877.

${ }^{43}$ El texto de este manuscrito permanece aún inédito; lo hemos colacionado sobre fotografías.

${ }^{44}$ M. A. Falconer Madan - H. H. E. Craster, A Summary Catalogue of Western Manuscripts in the Bodleian Library at Oxford (Oxford 1922) vol. II,1 pág. 51.
} 
a) En el uso del sustantivo superbia en lugar del adjetivo superbus, Valv y $\Lambda^{\mathrm{H}}$ coinciden con otros testimonios de $\mathbf{V}$, como son $\Sigma \Lambda^{\mathrm{L}} \Pi^{\mathrm{G}} \mathrm{AM} \Phi^{\mathrm{T}^{*} \mathrm{RP}} \Gamma^{\mathrm{A}} \Psi^{\mathrm{D}} \Omega^{\mathrm{MJ}}$ y SED-S rect $318 \mathrm{C}{ }^{45}$. Valv y $\Lambda^{\mathrm{H}}$ por otra parte, presentan un genitivo en donde los restantes testimonios tienen ablativo.

b) En el uso del genitivo, aunque con diferencia de tipo léxico, Valv y $\Lambda^{\mathrm{H}}$ coinciden con testimonios de $V L: 146$ (iniquitatis) y 7 $109 \mathrm{H}$ (iniuriae).

1 Mac 5,64: faustos; $L X X: \varepsilon \dot{v}(\varphi \eta \mu \circ \hat{v} \tau \varepsilon \varsigma)$

Valv coincide con $\Lambda^{\mathrm{H}}$, frente a la restante tradición textual de $\mathbf{V}$, que presenta fausta (adclamantes). La construcción adjetivo + participio $\left(\right.$ Valv y $\left.\Lambda^{\mathrm{H}}\right)$ en lugar de adjetivo / adverbio + participio (Vulg y testimonios de VL [bene acclamantes $94109146 \mathrm{H}$ y el palimpsesto de León ${ }^{46}$, Catedral 15,VL 67; bene clamantes 7]) se encuentra, con algunas diferencias, en una alusión de AN Apollonius 10,297,10 (recensión B): faustis adclamationibus ${ }^{47}$.

2 Mac 2,11: mundatum; $L X X: \beta \varepsilon \beta \rho \hat{\omega} \sigma \theta \alpha \iota$

Mundatum es la lectura de $\Lambda^{\mathrm{H}}$ y también de $\mathbf{V}$ : eo quod non sit mundatum.

2 Mac 12,15: uelociter; $L X X: \theta \eta \rho ı \omega \delta \omega \varsigma \varsigma$

La lectura uelociter está también atestiguada por $\Lambda^{\mathrm{H}}$, pero no se encuentra en ningún otro testimonio latino de los que hasta ahora han sido cotejados en 1-2 Mac. La lectura mayoritaria de la tradición textual latina es ferociter (V), a excepción del manuscrito de $V L 109$ (recensión $X$ ), que presenta uiriliter, y de $\Omega^{\mathrm{MS}}$, que presentan fortiter. En nuestra opinión, uelociter se puede explicar como una falta de copista producto de una confusión entre $(u)$ iriliter y $(f)$ erociter, o de dictado interno.

${ }^{45}$ Sedulio Escoto, De Rectoribus Christianis, ed. de J. P. Migne, PL 103, cols. 219-332.

${ }^{46}$ Para el palimpsesto hemos utilizado las transcripciones realizadas por B. Fischer que se conservan en el Vetus Latina Institut de Beuron (Alemania).

${ }^{47}$ Historia Apollonius Regis Tyrii, ed. de G. A. A. KortekA AS (Groninga 1984) págs. 278-411. 


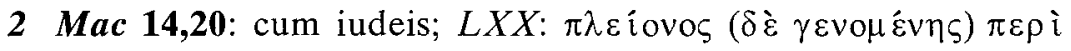

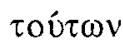

Valv presenta una clara falta de copista, iudeis en lugar de diu de his (V), que comparte con otros testimonios de Vulg, como son $\Lambda^{\mathrm{H}}$, y con algunas diferencias con $\Delta^{\mathrm{LB}}$ (de iudeis). La falta de copista iudeis de Valv y $\Lambda^{\mathrm{H}} \Delta^{\mathrm{LB}}$ se explica, a partir de diu de his, por la lectura palatalizada de $d i$ - en el dictado interno y una posterior armonización textual del contexto.

Del estudio de estas lecturas se obtiene una conclusión clara: Valv coincide especialmente con el manuscrito $\Lambda^{\mathrm{H}} \mathrm{y}$, más esporádicamente, con otros testimonios españoles, como los pertenecientes a la edición $\Delta$ :

a) Faltas de transmisión: 1 Mac 2,42 ad auxilium eorum Valv, con $\Lambda^{\mathrm{H}} ;$ cf. 146; 2 Mac 12,15 uelociter Valv, con $\Lambda^{\mathrm{H}} ; 2$ Mac 14,20 iudeis Valv, con $\Lambda^{\mathrm{H}} \Delta^{\mathrm{B}} ; c f . \Delta^{\mathrm{L}}$.

b) Variantes: 1 Mac 3,20 superbiae Valv, con $\Lambda^{\mathrm{H}}$; 1 Mac 5,64 faustos Valv, con $\Lambda^{\mathrm{H}}$.

Las coincidencias de Valv con $\Lambda^{\mathrm{H}}$ constituyen la gran mayoría; el hecho de que ambos testimonios compartan faltas tan llamativas puede hacernos suponer que, probablemente, $\Lambda^{\mathrm{H}}$ pudo haber sido copia de Valv. Estas lecturas comunes a Valv y $\Lambda^{\mathrm{H}}$ diferencian a este grupo del más antiguo, mejor y fiel testimonio textual de $\Lambda$ que conservamos en 1-2 Mac: el Codex Gothicus Legionensis $\left(\Lambda^{\mathrm{L}}\right)$ :

1 Mac 2,42 ad auxilium eorum Valv $\Lambda^{\mathrm{H}}$ ] Asideorum $\Lambda^{\mathrm{L}}$ con V

3,20 superbiae Valv $\Lambda^{\mathrm{H}}$ ] super(b)ia $\Lambda^{\mathrm{L}}$ con $\Sigma \Pi^{\mathrm{G}} \mathrm{AM}-$ $\Phi^{\mathrm{T}^{\star} \mathrm{RP}} \Gamma^{\mathrm{A}} \Psi^{\mathrm{D}} \Omega^{\mathrm{MJ}} \mathrm{SED}-\mathrm{S}$

5,64 faustos Valv $\Lambda^{\mathrm{H}}$ ] fausta $\Lambda^{\mathrm{L}}$ con $\mathrm{V}$

2 Mac 12,15 uelociter $V$ alv $\Lambda^{\mathrm{H}}$ ] ferociter $\Lambda^{\mathrm{L}}$ con $\mathbf{V}$

14,20 cum iudeis Valv $\Lambda^{\mathrm{H}}$ ] quamdiu de his $\Lambda^{\mathrm{L}}$, cf. $\mathbf{V}$ cum diu de his

En un caso, sin embargo, presentan plena coincidencia entre sí Valv y $\Lambda^{\mathrm{HL}}$, y además coinciden también con $\mathbf{V}$ :

2 Mac 2,11 mundatum Valv $\Lambda^{\mathrm{H}}$, con $\Lambda^{\mathrm{L}}$ y $\mathbf{V}$.

Además de la coincidencia de Valv y $\Lambda^{\mathrm{H}}$ entre sí, ambos testimonios presentan llamativas coincidencias con otros testimonios españoles de la misma época. Algunos ejemplos son: 
a) La edición $\Delta$, en 2 Mac 14,20. Esta edición fue realizada en el siglo VII en el norte de España (quizá Cardeña) ${ }^{48}$, aunque todos los manuscritos que la han transmitido datan del siglo $X^{49}$. La falta de transmisión iudeis podría encontrarse ya en el arquetipo $\Delta$ del siglo vII del que dependen $\Delta^{\mathrm{LBM}}$, y podría haber ejercido su influencia en testimonios de $\Lambda$ (como Valv y $\Lambda^{H}$ ), que es una de las ediciones españolas de $V u l g$ que presenta mayor mezcla textual.

b) El manuscrito de $V L 146$ (recensión $L$ ), en 1 Mac 2,42. La falta de copista ad auxilium eorum de Valv y $\Lambda^{\mathrm{H}}$ está relacionada con la que presenta el manuscrito 146 (in auxilia eorum); este manuscrito, en opinión de De Bruyne - B. Sodar, fue copiado sobre un modelo visigótico ${ }^{50}$.

Las conclusiones principales a las que hemos llegado, teniendo en cuenta las limitaciones que nos impone la escasez del texto de 1-2 Mac de Valv que se nos ha conservado, son las siguientes:

a) Como, en efecto, ya se había constatado respecto a Valv en general, también en nuestro estudio se confirma su pertenencia a la edición $\Lambda$.

b) En el ámbito de $\Lambda$, el texto de 1-2 Mac de Valv presenta una clara relación con el manuscrito $\Lambda^{\mathrm{H}}$; probablemente, Valv pudo ser el modelo sobre el que se copió el manuscrito $\Lambda^{\mathrm{H}}$.

c) En 1-2 Mac, Valv y $\Lambda^{\mathrm{H}}$ quizá constituían un subgrupo dentro de la edición $\Lambda$; así lo indica el hecho de que se diferencien ostensiblemente en las lecturas estudiadas del principal y más antiguo

\footnotetext{
${ }^{48}$ El texto $\Delta$ ha sido identificado especialmente en el Nuevo Testamento (cf. FISCHER «Bibelausgaben» pág. 79), pero DE BRUYNE - SODAR demostraron en su edición que este grupo de testimonios contiene una recensión particular de $1-2$ $M a c$ (sigla H). Los mss. que lo forman fueron copiados en el siglo X en el Norte de España, en letra minúscula visigótica, tomando como modelo una Vorlage que se remonta a la segunda mitad del siglo VII; sobre ella cf. FISCHER «Bibelausgaben» págs. 71-72; FREDE Ep. ad Ephesios pág. 20*, Ep. ad Philippenses pág. 19 y Ep. ad Thessalonicenses pág. 53; THIEle Sapientia pág. 60 y Ep. Catholicae pág. 28*; SCHUlZ-Flügel Canticum págs. 44-45; FröHLICH Ep. ad Corinthios I pág. 73; y EYMANN Ep. ad Romanos págs. 37-38.

${ }^{49}$ Estos manuscritos son: Catedral de León, 6, año 920 (sigla de Vulg, $\Delta^{\mathrm{L}}$ ); BAH, Aemilianensis 20 (sigla de $V u l g, \Delta^{\mathrm{M}}$ ); y SMB (sigla de Vulg, $\Delta^{\mathrm{B}}$ ). DE BRUYNE SODAR cotejaron los tres en su edición de Vulg como representantes de un texto español (sigla H); en Libri I-II Macchabaeorum se han cotejado sólo los dos primeros.

${ }^{50}$ Les anciennes pág. XII.
} 
representante de $\Lambda$, que es el Codex Gothicus Legionensis $\left(\Lambda^{\mathrm{L}}\right)$, que en estos casos está de acuerdo con $\mathbf{V}$.

d) Las lecturas de 1-2 Mac de Valv y $\Lambda^{\mathrm{H}}$ estudiadas no pertenecen al fondo original de la edición $\Lambda$, sino que constituyen innovaciones que han contribuido a empeorar su texto, a veces por influencia de otros testimonios del norte de España, como $\Delta$. Ningún testimonio de otras ediciones españolas, como la que se encuentra en el manuscrito Cavense (Archivo de la Badia I [14], copiado después del 850) ${ }^{51}$ o en la edición $\Sigma$ (sur de España, siglo VII) ${ }^{52}$ presentan coincidencias con Valv y $\Lambda^{\mathrm{H}}$.

${ }^{51}$ Sobre este manuscrito, cf. QUENTIN Memoire pág. 299, y Liber Genesis pág. XIV; T. Ayuso, «La Biblia Visigótica de la Cava dei Tirreni», EstBíb 14 (1955) págs. 49-65, 137-190 y 355-414, y 15 (1956) págs. 5-62; FrEDE Ep. ad Ephesios pág. 15*, Ep. ad Philippenses pág. 16, y Ep. ad Thessalonicenses pág. 35; THIELE Sapientia págs. 29-30, Sirach pág. 24, y Ep. Catholicae pág. 21*; SCHUlz-FLÜGEL Canticum págs. 26-27; FRÖHLlCH Ep. ad Corinthios I págs. 47-49; EYMANN Ep. ad Romanos pág. 27.

52 De esta edición se han cotejado en la edición Libri I-II Macchabaeorum dos manuscritos: UCM, 32 (sigla $\Sigma^{\mathrm{C}}$ ), y BNM, Vitr. 13-1 (Tol. 2-1), sigla $\Sigma^{\mathrm{T}}$. Ambos fueron copiados en el siglo X. Con la sigla genérica $\Sigma$ se designa una edición española del siglo VII, relacionada con Juan, obispo de Zaragoza (619-631) (FISCHER «Bibelausgaben» págs. 70-71; FREDE Ep. ad Thessalonicenses págs. 6869; THIELE Sapientia págs. 75-77, Sirach págs. 52-53, y Ep. Catholicae pág. 33*; SCHUlz-FlügEl Canticum págs. 53-54; FröHLICH Ep. ad Corinthios I págs. 98-99; y EYMANN Ep. ad Romanos pág. 51). 


\section{RESUMEN}

En este artículo se editan y estudian por primera vez dos testimonios fragmentarios de la Vulgata de 1-2 Macabeos de origen español. Ambos aportan luz a dos aspectos diferentes que atañen a la historia de la Vulgata latina en los siglos X y XI en España. El primero se trata del fragmento del Museo Episcopal de Vich (fragmento XIII/19, folio 8), del siglo XI, que contiene texto de 1 Macabeos 1,462,12, y es un ejemplo del tipo de texto de Vulgata de 1 Macabeos utilizado en Cataluña en el siglo XI. El otro testimonio es la perdida Biblia de Valvanera, de cuyo texto de Vulgata sólo se han conservado algunas lecturas copiadas a modo de glosas marginales por $\mathrm{H}$. del Castillo en 1561 en el incunable de El Escorial, 54.V.35.

\section{SUMMARY}

This study presents a first-time critical edition and analysis of two Spanish fragments of theVulgate 1-2 Macabees. Both readings shed new light on two different aspects dealing with the tenth- and eleventh-century Vulgate Latin version of the Bible in Spain. The first fragment dates from the eleventh century, and is part of the Vich Episcopal Museum collection (fragment XIII/19, fol. 8). A fragmentary text from 1 Macabees 1,46-2,12 is an example of the type of Vulgate text employed in eleventh-century Catalonia. The second fragment derives from the lost Valvanera Bible, parts of the Vulgate text of which have been conserved in sparse marginal glosses copied by $\mathrm{H}$. del Castillo in 1571. These glosses are located in the Escorial incunabulum 54.V.35. 